\title{
EL CONCEPTO DE MEMORIA SOCIAL COMO PROBLEMA PARA LA TEORÍA DE SISTEMAS SOCIALES
}

THE CONCEPT OF SOCIAL MEMORY AS A PROBLEM FOR SOCIAL SYSTEM THEORY

Mg. Santiago Calise (c_santiago_g2000@yahoo.com.ar) Instituto de Investigaciones Gino Germani, CONICET (Buenos Aires, Argentina)

\begin{abstract}
The concept of social memory is between those categories, which acquired more relevance in Niklas Luhmann's theoretic production in the last years of his life. This concept is largely inspired in the memory function depicted by the English mathematician George Spencer Brown. Social memory tries to account for the own memory performance of society, which is independent from the memories of the psychic and neurophysiologic systems. This paper deals critically with this topic, focusing the attention on the operativity of the social memory, in order to observe to which questions it can answer. In addition, it will be carefully analyzed how Luhmann utilizes the Spencer Brown's oscillator and memory functions, so to evaluate the consistency of Luhmann's decision to include these concepts in his theory. It will also be of great importance to estimate if the German sociologist develops a theory of social memory with the capacity to get rid of the storage metaphor, as he expected to have done with this formulation.
\end{abstract}

Key words: memory function, oscillator function, George Spencer Brown, semantic, self-descriptions.

\section{Resumen}

El concepto de memoria social es una de las categorías que más relevancia adquirió en la producción teórica de Niklas Luhmann en los últimos años de su vida. Tal concepto se encuentra, en una buena parte, inspirado en la memory fuction descripta por el matemático inglés George Spencer Brown. La memoria social pretende dar cuenta de un rendimiento de memoria propio de la sociedad, independiente de las memorias de los sistemas psíquicos y neurofisiológicos. En este trabajo se abordará el tema críticamente, poniendo atención en la operatividad de la memoria social, para poder observar a qué preguntas responde. Además, se analizará detenidamente el tipo de apropiación que hace Luhmann de la función de oscilador y memoria tomada de Spencer Brown, evaluando la consistencia de tal decisión. También será de suma importancia estimar si el sociólogo alemán arriba a una teoría de la memoria social capaz de desprenderse de la metáfora del almacén o depósito, tal como él dice haberlo hecho al proponer esta formulación.

Palabras clave: función memoria, función de oscilador, George Spencer Brown, semántica, autodescripciones. 


\section{Introducción}

El texto que se presenta a continuación (1) tiene la intención de avanzar en la crítica del concepto de memoria social propuesto por Luhmann, en el contexto de su teoría de sistemas. En esta ocasión, la investigación se centrará en la definición dada por el autor alemán respecto del concepto, sin llegar a explorar las implicancias empíricas que esta categoría pueda llegar a tener. Parte de esta tarea se concentrará en iluminar la relación entre las formulaciones de Spencer Brown y el uso que Luhmann hace de ellas. No obstante, en esta instancia no se avanzará hacia una crítica -tan necesaria, por otra parte- de la utilización de las Laws of Form dentro de la teoría de sistemas sociales, en general. Para ello, el lector interesado puede remitirse al texto de Boris Hennig "Luhmann und die Formale Mathematik" (2000), que es uno de los pocos dedicados a esta cuestión. En este sentido, el siguiente trabajo, en parte, puede ser leído como una contribución en esa dirección.

Cabe aclarar que Luhmann mismo no indica de manera clara y sistemática en qué modo introduce en su armado teórico los conceptos specerbrownianos, cosa que probablemente haya hecho con muy pocos conceptos. Si se busca un texto donde se intente esta indicación de la que se hablaba, probablemente "Frauen, Männer und George Spencer Brown" (1988) sea el trabajo donde, más explícitamente, Luhmann trabaje con la relación entre teoría de sistemas y las leyes de la forma. Al mismo tiempo, el texto es de una muy alta complejidad, debido al uso de esta teoría matemática, pero, por otro lado, las críticas direccionadas contra el feminismo y los movimientos feministas son claramente obsoletas y simplistas, más orientadas a la mera polémica que a la discusión sociológica. En efecto, el texto ignora buena parte de los avances teóricos del mencionado campo de estudios, pareciendo sostenerse únicamente en la gran autoridad teórica y académica del propio autor. No obstante, un punto importante que deja este texto es el distanciamiento que toma Luhmann respecto de un problema puntual, el de la distinción entre mujeres y hombres. Según el matemático inglés, ésta es una distinción que no distingue, sino donde ambos lados de la distinción tienden a fusionarse. Naturalmente que esto lo dice en un libro más poético-filosófico, que científico (Keys 1974). Luhmann explícitamente se aleja de esta propuesta y de sus implicancias filosóficas subyacentes. Importante sería saber si este procedimiento debe extenderse por completo a todos los usos de las Laws of Form que Luhmann realiza y si realmente es posible solucionar el problema tan fácilmente, o sea, trabajar con un mero cálculo simplificado de las distinciones, eliminando buena parte de los presupuestos filosóficos y epistemológicos que le dieron origen y lo sostienen.

Respecto del concepto de memoria social, tal como se lo pretende iluminar en este trabajo, o sea, en el diálogo que establece con la teoría de Spencer Brown, no se ha encontrado literatura abocada a esta tarea. No obstante, cabe citar el destacado trabajo de Elena Esposito (2002), centralmente dedicado al estudio de la evolución de la memoria social, prestando específica atención a las cuestiones de semántica. Lamentablemente, la socióloga italiana ha omitido la discusión que se quiere abrir en este estudio con la matemática de las formas.

Se comenzará por hacer una breve presentación de las formulaciones del matemático inglés, para poder observar su relación con los conceptos elaborados por Luhmann, respecto de la memoria social. Luego se avanzará en la exégesis de los textos del sociólogo, con el fin de poder arribar hasta las definiciones y poder evaluarlas. 


\section{La "memory function" en las leyes de la forma}

Spencer Brown comienza, explícitamente, dando por sobreentendidos los conceptos de distinción e indicación. La distinción es concebida como continencia perfecta, por lo cual, para poder pasar del otro lado de una frontera, es necesario cruzar esta última. Los dos primeros axiomas de su matemática serán la "law of calling", según la cual "the value of a call made again is the value of the call" (1972:1) y la "law of crossing", por la cual "the value of a crossing made again is not the value of the crossing" (1972:2). A partir de estos fundamentos, se erigirá la "primary arithmetic", basada en las famosas expresiones:

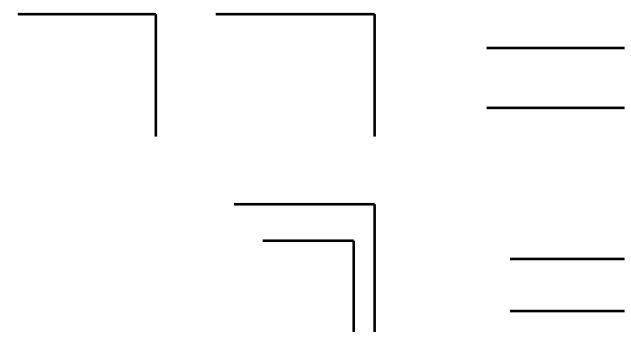

En este espacio no se procederá a explicitar los pasos que llevan de la aritmética al álgebra, sino que se pasará directamente a los conceptos relevantes para los fines de este trabajo. Las funciones de memoria y de oscilador, en la matemática de Spencer Brown, son propiedades de las ecuaciones de segundo o mayor grado, o sea, de aquellas que tienen una o más re-entries. El problema de la re-entry aparece cuando uno se encuentra con ecuaciones que no admiten una solución aritmética, o sea, que no pueden decidirse por medio de un razonamiento de primer grado, por lo cual se hace necesario admitir un imaginary state, que es un estado que cambia en el tiempo, pero no en el espacio, por lo cual se entra en un estado de tiempo diferente, sin dejar el precedente estado en el espacio. En este sentido es que los valores de la ecuación comienzan a oscilar. De esta manera, las variables empiezan a alternar sus valores de marked a unmarked. En relación con esto, la función memoria (memory function) implica que el valor actual de la función dependería del valor pasado asumido por sus variables, valores que no pueden ser otros más que marked y unmarked. Por otra parte, para que una función comience a oscilar, Spencer Brown sostiene que es necesario suponer la existencia de un túnel que conecte marked y unmarked spaces, de manera que se dé una transmisión de uno al otro.

Quizás, anticipándose a posibles usos o refutaciones de estas ideas, el matemático inglés se encarga de aclarar, en las notas, que la memoria de los animales no funciona de la manera como fue explicada en el ensayo. Sin embargo, esta idea sí ha servido en el desarrollo de circuitos eléctricos y de computadoras, y, posiblemente, pueda ser vista como "a precursor of the more complicated and varied forms of memory and information in man and the higher animals. We can also regard other manifestations of the classical forms of physical or biological science in the same spirit" (1972:100). Metafóricamente hablando, puede entenderse que la propuesta de Spencer Brown implica pensar a la memoria en términos de "circuito" y no de "depósito". En este sentido, a la memoria del cerebro se la suele entender como producto de conexiones sinápticas, que, al disolverse el vínculo entre neuronas, se produce el desvanecimiento del recuerdo (Kandel 2001). Por su parte, Heinz von Foerster (2003a), en una conferencia que ya tiene unos cuarenta años, presenta dos formas usuales de pensar a la memoria neurofisiológica: aquella que la entiende como "almacenamiento y recuperación" ("storage and retrieval") y la que la comprende como "reconocimiento y recuerdo" ("recognition and recall"). Respecto de la primera, el físico austríaco señala que tal concepción 
implica pensar en una especie de inalterabilidad de la cualidad de lo que es almacenado, que luego puede ser recuperado. Pero si se entiende que el cerebro funciona de esta manera, se hace necesario presuponer la existencia de un pequeño demonio, provisto de capacidades cognitivas, que debería explorar este enorme depósito para extraer las informaciones necesarias.

Al momento de escribir Sistemas Sociales, Luhmann $(1984,1998)$ no había intentado introducir el concepto de memoria, bastándole los conceptos de "cultura" y "semántica". El primero de ellos indicaba una "provisión" de temas, mientras que el segundo representaba un almacenamiento con fines comunicativos. En ese momento, parecería ser que Luhmann no se sentía incómodo con la idea de "depósito". Será en textos posteriores, en donde también se multiplican las citas de Spencer Brown, donde el concepto de memoria haga su aparición.

\section{Re-entry y autodescripción}

Retornando al problema de la re-entry, en el capítulo 11 de Laws of Form, Spencer Brown comienza intentando demostrar que una demostración, valga la redundancia, radica en un número finito de pasos. Sin embargo, una expresión algebraica representa un número desconocido de cruces, lo que hace imposible contar pasos aritméticos. Esto se debe a que el estado actual de la ecuación podría estar precedido por un número desconocido de estados anteriores. Por lo tanto, se hace posible seguir calculando infinitamente, obteniendo como resultado que los valores asumidos por las variables de la ecuación en cuestión no serían los mismos que los de estas mismas variables en la ecuación inicial. No obstante, lo que se verifica es que, la parte que cruza en cada nivel de profundidad par (1), es idéntica a la expresión total. Por lo cual, en cada nivel de profundidad par, la expresión reentra en sí misma. Luego de aplicar la regla de la dominancia (ver 1972:56), se puede observar que esta ecuación puede tener dos soluciones. Consecuentemente, concluye Spencer Brown, desde un número ilimitado de pasos desde una expresión $e$, se puede arribar a una expresión $e^{\prime}$, que no está determinada de una sola manera. De esta manera, $e^{\prime}$ implica un nivel de indeterminación que no se puede resolver manteniendo fijo el valor de cada variable independiente.

En relación con el concepto de autodescripción, Luhmann señala lo siguiente: "Si el sistema de la sociedad no sólo produce la diferencia sistema / entorno, sino además se orienta por ella, tenemos aquí entonces el caso de una "re-entry" de la forma en la forma (de una distinción dentro de una distinción), que pone al sistema en un estado de "unresolvable indeterminacy". 'Unresolvable' quiere decir que las operaciones matemáticas normales de la aritmética y el álgebra ya no llevan a resultados inequívocos. El sistema -para ayudarse- requiere de números imaginarios o espacios imaginarios. En verdad éste no es un argumento que para la teoría de la sociedad evidencie cosa alguna, pero la inalcanzabilidad comunicativa de la sociedad (es decir, el fracaso de aquellas operaciones que reproducen al sistema) consta empíricamente como certeza. Y en su lugar existen construcciones imaginarias de la unidad del sistema que posibilitan la comunicación en la sociedad -aunque no con ella sino sobre ella. A tales construcciones denominaremos "autodescripciones" del sistema de la sociedad" (2007b: 687).

Trazando una analogía con las explicaciones de Spencer Brown, Luhmann equipara las operaciones comunicativas con las operaciones aritméticas, debido a que, con ninguna de ellas, puede resolverse la "unresolvable indeterminacy" que se manifiesta cuando la operación de re-entry se manifiesta. En el caso del cálculo de las distinciones, el problema será resuelto a través de valores imaginarios que cambian en el tiempo, como se ha visto previamente. Mientras que en el caso de los sistemas sociales, tal estado es solucionado a través de construcciones comunicativas "imaginarias", que representan la unidad del sistema. 
En la teoría de Luhmann, la posibilidad de poder distinguir entre un nivel meramente operativo, ciego podría agregarse-, se da gracias a la diferenciación entre operar y observar. Debido al hecho de que la sociedad es un sistema que observa y que se autoobserva, puede llegar a construir tales representaciones de su propia unidad. Unidad que es esencialmente inalcanzable, debido a que es siempre una parte de la sociedad la que intenta observar a la sociedad en su totalidad, por lo que, al observar, no puede incluirse en su observación. Por lo tanto, la observación se vuelve inevitablemente parcial. Consiguientemente, estas construcciones son imaginarias a raíz de que, pese a ser parciales, pretenden representar una totalidad, al tiempo que también lo son por desarrollarse solamente en el tiempo de la sucesión de eventos comunicativos observacionales. Para poder afirmar tal cosa debería poder hablarse de un desdoblamiento del tiempo, en un tiempo operacional y un tiempo observacional, aunque la distinción se vuelve un tanto lábil, puesto que en ambos casos se habla de operaciones comunicativas que, pese a ocurrir en un nivel distinto, contribuyen a la autopoiesis del mismo sistema.

\section{La memoria social}

Memoria y cultura

Es a partir de la re-entry, que permite construir autodescripciones y conformar una semántica, que, para Luhmann, se hace necesario el concepto de memoria, en analogía con las formulaciones de Spencer Brown. Sin embargo, este detalle no es menor, ya que el concepto de memoria social propuesto por Luhmann se volverá incapaz de dar respuesta al problema más profundo de la posible e inexorable dependencia mnemónica de la sociedad respecto de los sistemas psíquicos (si es que éstos tienen una), o de los sistemas neurofisiológicos. Con esto se quiere sostener que, si en una situación donde alter y ego participan de la comunicación -siendo ambos sistemas psíquicos-, la comunicación deberá confiar completamente en la memoria a corto plazo de ambos cerebros, si pretende que ambos sistemas no se olviden al poco tiempo de lo que habían dicho hace pocos instantes. En esta situación de interacción, al haber una clara dependencia mnemónica, podría estarse al filo de una dependencia informativa también, recordando que la información es siempre, para Luhmann, una selección de la operación comunicativa.

Dejando de lado este problema, que supera los objetivos de este trabajo, Luhmann opta por una concepción meramente culturalista de la memoria social, postulando que la cultura es la memoria de la sociedad, en cuanto sistema social (1997b). No obstante, pretende distanciarse de la idea de "memoria colectiva", debido a que, para él, la memoria social no se establece por los rendimientos de memoria de los sistemas psíquicos, puesto que los primeros son demasiado diversos como para conformar una memoria social. Apartando estas cuestiones, Luhmann afirma que la memoria social aparece como producto colateral de la forma que asume la operación basal autopoiética, por la que el sistema se reproduce. Este último se hace dependiente de tal situación, debido a que las operaciones del sistema se producen bajo la forma de acontecimientos (1997b). Más allá de esta definición respecto de su origen, no queda claro qué rol ocupa este concepto de memoria dentro de la sociedad. En varias ocasiones (Luhmann 1995, 1997a, 2007b, 2008) Luhmann la califica como "función", y, en muchas de ellas, citando a Spencer Brown. Por otra parte, al hablar de la sociedad funcionalmente diferenciada, el autor entenderá que en ella, cada sistema funcional está dotado de una memoria (2000a, 2007b). Normalmente, en la teoría de Luhmann, cada sistema cumple una función, sin embargo, resulta difícil, si no imposible, sostener que la memoria es un sistema. La hipótesis más aceptable respecto de este problema sería suponer que Luhmann utiliza el sustantivo "función" solamente como mera referencia al texto de Spencer Brown, sin otorgarle a esta palabra la carga semántica que ella conlleva al 
interior de la teoría que el primero de los autores citados elaboró. Más allá de este problema de precisión conceptual, el sociólogo entiende que la función principal de la memoria es la de olvidar, evitando que el sistema se bloquee por la coagulación de resultados anteriores, dejando libres las capacidades para recibir nueva información. Por lo tanto, la memoria trabajaría distinguiendo entre olvido y recuerdo, indicando este último lado sólo en situaciones muy particulares, o sea que se recuerda sólo aquello que se volverá a repetir, favoreciéndose ciertas condensaciones. De esta manera se logra que el sistema observe al entorno y termine por tratarlo como idéntico (1997b). Por otro lado, Luhmann también le asigna a la memoria la tarea de verificar "la consistencia de todas las operaciones que suceden en vista de lo que el sistema construye como realidad", garantizando "Ios límites de las posibles pruebas de consistencia" (2007b: 457).

Retornando sobre la distinción entre olvido y recuerdo, Luhmann asevera que la repetición permite, a la vez, olvidar y recordar. Lo primero ocurre al dejarse de lado la situación previa al suceso descripto y la sorpresa producto de su novedad, mientras que lo que se recordaría sería la referencia o las palabras que la designan, en cuanto hecho familiar, que no causa sorpresa alguna. El ejemplo que da el autor a este respecto es la nonecesidad de tener que conocer la historia de la partida, cuando uno se dispone a solucionar un problema de ajedrez de un diario. No obstante, Luhmann destaca que una charla no puede estarse olvidando a cada instante, aunque no provee una respuesta a cómo esto no sucede. De todas maneras, el sociólogo alemán cree que basta con que el presente sea visto como un pasado coagulado, siempre que haya suficientes "identidades" que permitan el enlace entre pasado olvidado y futuro. Tales identidades pueden llegar a convertirse excepcionalmente en "objetos" o "valores propios". Heinz von Foerster (2003b) nota como, desde una epistemología (circular y cerrada) que incluye un observador, los "objetos" -o sea, lo que ve una epistemología (linear y abierta) que excluye al observador-, son vistos como "símbolos para comportamientos estables" (tokens for stable behaviors). En su texto, el físico austríaco muestra como ciertas funciones matemáticas (y no-matemáticas) desarrollan "valores-propios", que representan posiciones estables que se logran al aplicar recursivamente los resultados de las operaciones a las propias operaciones. Vistos ontológicamente, estos valores-propios son estables, son los “objetos" que el sistema, a partir de su red de operaciones, ha producido. Por su parte, Luhmann (2002) indica que los sistemas que procesan sentido trabajan por medio de las operaciones de condensación y confirmación de distinciones, generando, a la vez, la diferencia entre lo actual y lo posible, constitutiva del medio sentido.

Compensación y condensación son los dos sentidos en los que es posible leer el axioma 1. "The law of calling" de Spencer Brown. Tal axioma dice: "The value of a call made again is the value of the call. That is to say, if a name is called and then is called again, the value indicated by the two calls taken together is the value indicated by one of them. That is to say, for any name, to recall is to call" (1972: 1) Gráficamente, esto es expresado de la siguiente manera: >>=>. Si se lee de izquierda a derecha, se está frente a la condensación, mientras que si se lo lee en el otro sentido, se lo interpreta como una compensación.

Lo que permite el juego entre condensación y confirmación es que se formen identidades compatibles con diferentes situaciones y que se designe un cierto campo de posibilidades. Las formas que asumen estas identidades son la de cosa y concepto. En este sentido, los valores propios que genera el sistema en su operar recursivo, serían reconocibles como "identidades". Esto se debe a que, al sistema, tanto las cosas como los conceptos le serían constantemente reconfirmados en la condición condensada por una operación previa.

En relación con los valores propios de los sistemas sociales, en algunas ocasiones (2000b, 2008) Luhmann 
destaca que los temas cumplirían tal función. En Sistemas Sociales, los temas solucionan el problema de cómo hacer para que la comunicación se convierta en un proceso, ya que éstos permiten la ordenación de las relaciones entre las comunicaciones. Como se había mencionado anteriormente, en ese momento Luhmann concebía a la cultura y la semántica como almacenes de temas. En La sociedad de la sociedad, se equipara la distinción entre temas y aportaciones con la de estructuras / operaciones, afirmando que, de esta manera, la memoria se articula por temas.

Retornando sobre el tema de la cultura, cabe realizar una pequeña digresión a este propósito. Para Luhmann, el concepto de cultura surge en Europa hacia la mitad del siglo XVIII, debido a que muchos campos comienzan a observar a través de la observación de segundo orden. Esto aparece, justamente, en un momento donde el primado de la diferenciación estamental se pierde, al tiempo que los horizontes históricos y regionales se expanden hasta alcanzar la totalidad del mundo. En este contexto, emerge un fuerte interés por la comparación (1997b). En este sentido, Luhmann deja atrás la primera formulación del concepto, presente en Sistemas Sociales, donde la cultura es pensada como un acervo de temas listos para la comunicación. Al concebir a la cultura en sentido histórico, se debe renunciar a la pretensión parsonsiana de considerarla como uno de los componentes de la acción, o la visión bourdieusiana, que la ve como capital, como acumulación. Por lo tanto, la cultura se definirá como aquella esfera en donde todos los testimonios de la actividad humana son registrados por segunda vez, no por su posible utilidad, sino para ser comparados con otros testimonios (2005). Luhmann no es muy benévolo con este concepto, ya que entiende que haber observado al arte y a la religión a través de este tipo de observación, trajo consecuencias desastrosas para estos dos sistemas. En cuanto al arte, llevó a que ya no sea posible observar a las obras de arte como tales, debido a que la cultura misma implica el gesto de querer compararlo todo. Pero además, las mismas obras de arte ya son producidas solamente con meros intereses comparativos, el arte se ha vuelto una constante búsqueda de observar re-entries. Por el lado de la religión, al observarse toda comunicación como contingente, la religión se volverá una mera cuestión de opción, al tiempo que la teología -en cuanto forma reflexiva del sistema- se vio discutida en su fundamento, perdiendo su autoridad (2007a).

Pese a todo lo anterior, el altanero concepto de cultura, que parece poder criticar y comparar todo, no puede autoobservarse. La técnica de la comparación no aclara nunca desde dónde el observador se detiene a observar con el fin de comparar, por lo cual debe recurrir a la semántica decimonónica -aun no superada-, de los motivos, estructuras y funciones latentes, que conducen la acción desde lo inconsciente. Por otro lado, la cultura, al duplicarlo todo, ha conducido hacia un culto penoso de la inmediatez, la autenticidad y la identidad, lo cual, es a su vez paradójico, porque estos son conceptos culturales no pueden mantener la propia promesa.

Por consiguiente, la memoria así entendida implica, fundamentalmente, un modo específico de observar (la observación de segundo orden), que conlleva la comparación. Centrándose en la observación y la comparación, parece dejarse de lado, o, al menos pierde importancia, la función de almacén semántico que tenía el concepto de cultura en formulaciones anteriores. No obstante, este concepto de cultura, producto de un análisis socio-histórico de la diferenciación en sistemas funcionales, tiene un origen bien distinto respecto del concepto de memoria que aquí se viene analizando. Esto significa que la equiparación de un concepto con otro no es algo evidente, sino que requiere de análisis ulterior. Hacia el final de la próxima sección se volverá a retornar sobre este acoplamiento entre memoria y cultura. 
Memoria y oscilación

Respecto de la temporalidad del sistema, la memoria no es ningún desenterrar cosas del pasado, su operatividad debe ocurrir completamente en el presente, que es siempre la distinción entre pasado y futuro. La memoria administra esta distinción y "controla la resistencia de las operaciones del sistema contra las operaciones del sistema", vigilando así "desde qué realidad el sistema mira hacia el futuro" (2007b:459). Especificando un poco más este argumento, Luhmann afirma, desorientando al lector, que la "función de transferencia" (Transferfunktion) de la memoria se refiere a distinciones. Por consiguiente, se opera del lado de lo designado exitosamente y se olvida el otro, o se recuerda una forma de dos lados y se olvida respecto de qué se distinguía esta forma.

En sintonía con lo expuesto al inicio de este trabajo, sobre las ideas de Spencer Brown, Luhmann retoma los conceptos de "memory function" y oscilación (2008). Al igual que el matemático inglés, Luhmann sostiene que para poder afrontar la situación de "unresolvable indeterminacy" producida por la re-entry, es necesario utilizar esta dupla categorial. Como ya se había visto, la función memoria lo que hace es presentar al presente como resultado del pasado o como producto de la distinción entre olvidar y recordar. Por lo tanto, esta función acompaña necesariamente a todas las operaciones de los sistemas que observan. Por el otro lado, aparece la función de oscilador, que permitiría, ante el despliegue de una distinción durante la observación, que el sistema pueda observar también la posibilidad de cruzar el límite y moverse de un lado a otro. De esta manera, lo que se gana es un horizonte de pasado para el presente, aliviando el costo de tener que ver toda situación actual como completamente nueva y tener que comenzar desde el principio. Al mismo tiempo, la función de oscilador deja un futuro abierto, en el sentido de que todo puede ser distinto, pero siempre dependiendo de la distinción que se esté utilizando. Ésta, al incluir aquello que se excluye, indica también aquello que podría ser de otra manera. Consecuentemente, el pasado puede ser visto como lo necesario y el futuro como lo contingente.

Como puede notarse, Luhmann traza claramente una analogía entre los conceptos de memoria y oscilación de la matemática de Spencer Brown y el reino de los sistemas sociales. Previamente se había visto que Spencer Brown postula la existencia de un túnel que conecta el lado marcado y el no-marcado, de manera que pueda trasmitirse un impulso (pulse) de un lado a otro. Según señala el autor, tal formulación ha sido de utilidad para la construcción de circuitos. Claramente, en el caso de este tipo de cálculo, los valores asumidos por las variables son sólo dos, mientras que las variables algebraicas mismas no tienen un contenido semántico específico. Por otro lado, la autorreferencia que se produce a través de la re-entry, en el caso de la matemática specer-browniana, puede ser una autorreferencia puramente vacía, sin implicar contenidos específicos, como en el caso de la reentrada de las formas estructuradas lingüísticamente, propias de los sistemas comunicativos. Para las leyes de la forma, este par de conceptos es muy sencillo: por un lado, la memoria recuerda cual fue el último valor asumido; por el otro, la oscilación alterna marked y unmarked state. Trasladar estos conceptos a la teoría de los sistemas sociales no es tarea fácil, puesto que las diferencias entre ambos ámbitos son enormes. En el contexto de los sistemas de comunicación, aquello que oscila son las distinciones, producto de la condensación de comunicaciones realizadas en el pasado y que han llegado a convertirse en puntos de vista dignos de ser recordados. Esto significa que la memoria no le ofrece al sistema la posibilidad de recordar la última unidad comunicativa, entendiendo que cada unidad estaría conformada por la triple selectividad, y que el proceso comunicativo sería el resultado de la unión, a través de la selección de la aceptación, de estas unidades mínimas. La memoria social así comprendida, es una memoria semántica. 
Respecto del funcionamiento de estos conceptos, a continuación se transcribe un pasaje de difícil interpretación, donde Luhmann intenta aclarar el funcionamiento de las distinciones en relación con las funciones de memoria y oscilación: "Aclarar este trasfondo de las distinciones con cuya ayuda un sistema observa, vuelve comprensible cómo la memoria que allí coopera distingue y enlaza pasado y futuro. Mientras en el ámbito de lo pasado la distinción misma queda sin marcarse (lo cual -como lo hemos dichotambién vale para la distinción de distinciones), en el ámbito que se desempeña como futuro se utiliza la distinción para posibilitar una oscilación, un cruce de la frontera interior (3). Si hasta ahora -por ejemploen los asuntos fuera de la circunscripción domésticas (asuntos 'políticos') siempre se ha hablado explícitamente o implícitamente sólo de hombres, puede diseñarse un futuro, en el que se recuerde, que hombres y mujeres se distinguen, y que, dentro de esta distinción se da la posibilidad de cruzar la frontera y volver de regreso, es decir, es posible oscilar. Entonces, en el presente la memoria se desempeña como el recuerdo de la distinción o como el reemplazo de la distinción hombre / unmarked por la de hombre / mujer; o como el alejamiento de esta distinción del contexto de la distinción polis / oikos (léase 'comunidad de hembras' en Platón) para ganar un pasado que ponga a disposición, para el futuro, un margen para oscilar" (2007b:460).

Lo que parece decir este párrafo es que una distinción permanece en el ámbito de la memoria, del pasado, sin ser marcada; mientras que del lado de la oscilación, del futuro, esta misma es activada en el ir y venir de un lado al otro. Retornando una vez más sobre Spencer Brown, para el matemático inglés la memoria era solamente recordar el último estado que fue marcado, pero aquí Luhmann señala tres formas en las que la memoria funciona. La más sencilla y la única que tendría un parecido con lo dicho por Spencer Brown, es la de recordar una distinción. La segunda es la de sustituir una distinción por otra (en el ejemplo se sustituiría, dentro de la forma "participantes en la actividad política", la distinción hombre / unmarked, por la que agrega a la mujer dentro de la forma). La tercera sería la de separar la distinción respecto del contexto estructural-social que la produjo. En este punto, quizás, más que ganar un pasado -como dice el autor-, se tendría que perder el pasado para poder destrabar la distinción respecto de la otra distinción que hace referencia a la estructura social y, de esta manera, poder utilizarla sin referencia a un contexto y permitir la oscilación.

Las dos últimas formas de trabajar de la memoria implican una selectividad muy especial, que supera el mero recordar. Parecería más acorde a la teoría misma y al concepto de memoria, suponer que esta última sólo cumple con la primera forma operativa y las otras dos, que implican una modificación sustancial en la distinción recordada, sean producto de la operación comunicativa en cuestión, que elige cómo presentar la distinción que va a utilizar. Si nos atenemos a las palabras de Luhmann, la memoria es una "función" y no una forma de operar. La memoria no realiza ninguna "operación", puesto que si eso hiciese, trazaría continuamente distinciones, o sea, pondría marcas de distinción entre dos estados, tal como Spencer Brown y Luhmann estarían dispuestos a entender tal concepto. Si la memoria operase comunicativamente, no podría distinguirse entre la memoria misma y la operación comunicativa.

Por otra parte, otro problema que plantea esta formulación, es la pregunta por el cómo se hacen presentes estas distinciones a la memoria. Si, como pretende Luhmann, su teoría de la memoria social se deshace de la metáfora del almacén, no queda claro cómo es posible que la memoria haga presentes estados pasados que son recordados. Recurrir a Spencer Brown, en cuanto este autor propone una manera de entender la memoria de forma recursiva, en el contexto de un circuito cerrado, no soluciona el problema. No lo resuelve, en primer lugar, porque las redes que teje la comunicación no son equiparables a los circuitos 
diseñados por el matemático inglés. En principio, estos trabajan con variables algebraicas, que distinguen sólo dos estados, y que no tienen más "materialidad" que la de la inscripción matemática.

Con esto se quiere decir que la matemática no es un sistema que opere empíricamente, y los circuitos que ésta diseña tampoco tienen una existencia operativa empírica. Que estos conceptos matemáticos sirvan para construir circuitos eléctricos, o de otra naturaleza, no le otorga mayor veracidad empírica a los dichos del matemático. En principio, porque el impulso transmitido sería un impulso eléctrico, y no el impulso del que habla Spencer Brown, que no parecería tener una naturaleza empírica. El matemático inglés, a través de su libro, parece querer develar una especie de lenguaje arquetípico -equiparable a las investigaciones cabalísticas que buscaban descifrar el nombre de Dios y las palabras utilizadas por éste a la hora de crear el mundo- $y$, de esta manera, parece pretender ver en ciertos órdenes de realidad la reproducción más o menos precisa de este orden arquetípico. De todas maneras, el mismo Spencer Brown fue cauto a la hora de trazar arriesgadas analogías, como se ha visto más arriba, a propósito de la posible relación entre memory function y la memoria animal, donde se destacaba que la primera podría ser un "precursor" de la segunda.

En este sentido, el matemático nota que "we do not imagine the wave train emitted by an excited finite echelon to be exactly like the wave train emitted form an excited particle. For one thing the wave form from an echelon is square, and for another it is emitted without energy. (We should need, I guess, to make at least one more departure from the form before arriving at a conception of energy on these lines.) What we see in the forms of expression at this stage, although recognizable, might be considered as simplified precursors of what we take, in physical science, to be the real thing" (1972:100-101). Más adelante, Spencer Brown se quejará de que, especialmente en la actualidad, la existencia es vista como fuente de realidad, cuando ésta "is seen to be highly peripheral and, as such, especially corrupt (in the formal sense) and vulnerable" (1972:101). La "existencia" es entendida, por el autor, en su sentido etimológico, como un estar afuera. Por consiguiente, agrega el matemático, la verdad sería un concepto más central. En relación con esto, es que Spencer Brown enuncia su famosa máxima hermética, por la cual: "we must abandon existence to truth, truth to indication, indication to form, and form to void, that has so held up the development of logic and its mathematics" (1972: 101). Aquí parecería afirmarse que la realidad está ordenada de una forma jerárquica, donde, en el mayor nivel de profundidad (para usar el lenguaje del autor), se encontraría la nada, de la cual van emanando los demás niveles. En esta clave budista es que parecería deber entenderse la arriba citada "law of crossing", por la cual, la nada tendría un poder creativo. Por otra parte, el concepto de "existencia" parecería remitir también al Pseudo Dionisio Areopagita (1981), y es por ello que antes se utilizó, no casualmente, la palabra emanación. En efecto, para el Pseudo Dionisio, Dios tiene la tendencia a salirse de sí mismo, a desbordarse, a través de la emanación, mientras que los seres creados tendrían la tendencia a superarse a sí mismos por medio de la actividad espiritual. Este "salirse de sí mismo" que crea las almas humanas es el "éxtasis" divino, y, de manera opuesta, las almas, a través de su éxtasis, deberían volver a Dios. Esto muestra el parentesco entre el "éxtasis" y la "existencia", de la cual hablaba Spencer Brown. Lo que existe tendría su ser fuera de sí, por eso debe retornar, que es lo que propone el autor en la última cita arriba reportada.

Volviendo al argumento, en este caso no hay ningún sistema omniabarcador que tenga una memoria de todas las operaciones matemáticas, sino que la posibilidad de recordar se da solamente dentro del circuito propuesto y nunca más allá de él. Por su parte, la comunicación no funciona produciendo redes tan simples y con variables tan pobres en información. Aun si se piensa en la forma más simple de comunicación, la interacción entre dos personas, sería demasiado reduccionista pensar tal situación como un circuito cerrado. 
De todas maneras, con relación a la memoria social, si alguno de los interactuantes hiciese referencia a alguna distinción conocida como resultado de una comunicación pasada, habría que integrar a esa otra comunicación en el circuito, que a su vez involucraría otras, derivando en una red inabarcable. Al mismo tiempo, si se acepta la precedente demostración, quedaría por preguntarse en qué espacio es que una distinción comienza a oscilar. El túnel es el canal a través del cual una ecuación transmite alternadamente en el tiempo, un impulso. El problema adicional que presenta esta metáfora spencerbrowniana, es que chocaría con la intención original de la teoría comunicativa formulada por Luhmann, ya que la figura del túnel implica que una información se transmite por un canal, al estilo de la teoría matemática de la comunicación de Shannon. El sociólogo alemán, precisamente, intenta romper con esta formulación, negando la metáfora de la transmisión y deshaciendo la unidireccionalidad del acto comunicativo. Asimismo, ningún participante en la comunicación recibe, alternadamente, una distinción. Se puede recibir una propuesta comunicativa que utilice un lado de una distinción y luego recibir otra o contestar con otra que llame en causa el otro lado de esa misma distinción antes mencionada. Sin embargo, resulta complicado, si no es absolutamente imposible, pensar en el oscilar de la distinción.

Como resultado, la utilización de los conceptos de Spencer Brown resultan ser un obstáculo, ya que no llegan a generar, ni siquiera de manera metafórica, una verdadera clarificación del concepto de memoria. No obstante, aun si se aceptase este uso, por la misma naturaleza de la teoría de sistemas sociales propuesta por Luhmann, resulta dificultoso sostener que la memoria social no es un depósito. Si se piensa que la memoria social contiene distinciones, estas distinciones no van a ponerse en marcha si no es a través de una operación comunicativa. Para poder recordar la distinción entre polis / oikos, propuesta por Luhmann -sin recurrir a la memoria neurofisiológica-, el participante en la comunicación debe ir a leer un texto o escuchar alguna comunicación que la refresque, pero, esto último, nos volvería a llevar, en algún momento, a un texto. ¿Pero, esto significa que el texto, en última instancia, es la memoria misma de la sociedad? Las respuestas de Luhmann son contradictorias. Por un lado, en La ciencia de la sociedad, el autor destaca que "los textos mismos todavía no son memoria operativa, fungiendo actualmente. Únicamente son artefactos, posibles de la prueba memorativa de consistencia” (1996:118). Por el contrario, en Organisation und Entscheidung, texto editado póstumamente, Luhmann afirma que "los textos son la memoria de los sistemas sociales, sin importar si están fijados por escrito o si son solamente transmitidos oralmente" (2000b:418) [La traducción del alemán es mía]. Pese a que se puede pensar que este último texto no pudo ser revisado por el mismo autor antes de salir a la luz, esta posición resulta bastante difícil de sostener, si es que se intenta escapar a la metáfora del depósito. En La sociedad de la sociedad, el autor se encarga de aclarar que la escritura permite la diferenciación de una específica memoria social, sin por ello llegar a aseverar que escritura y memoria son términos intercambiables. El lector puede conservar sus dudas respecto de esta polémica. De todas maneras, por lo visto hasta aquí, no hay forma de explicar cómo es que los recuerdos se hacen presentes. Si, en última instancia, siempre se haría referencia a un texto para poder recordar distinciones, entonces se debe ir a buscar el texto y leerlo. Pero si hay un "ir a buscar" (en cuanto recordar y no como acto de investigación), es difícil suponer que uno puede ir a buscar lo que no conoce o no sabe -a menos que se presuponga alguna especie de motivación inconsciente-, por consiguiente, será irremediable recurrir a la memoria psíquica o neurofisiológica. La única manera de poder refutar esto último es, inspirados en von Foerster, suponer la existencia de un pequeño demonio en los sistemas sociales, que introduzca las distinciones necesarias, sin que los participantes se percaten. Por consiguiente, parecería ser que la única manera de salvar la autonomía de la memoria social sería tratando de identificar quién o qué es este pequeño demonio. 
Para concluir, cabe retornar sobre la polémica identificación entre cultura y memoria social. En principio, la cultura ha quedado definida como un observar de segundo orden que implica comparación. Éste redescribe las descripciones de la vida cotidiana, duplicando el sentido de los objetos, para copiarlo en un plano de segundo orden (2007a). Este trabajo es realizado desde algún sistema (el del arte o de la religión, por citar los ejemplos ya tratados), lo cual significa que la cultura no es ningún sistema que observa. Por su parte, el concepto de memoria social entraña la necesidad de olvidar y recordar. Si cultura y memoria social se identifican, esta selectividad de olvidar / recordar debe conllevar una observación de segundo orden, cosa que es posible aceptar. Sin embargo, esto supone pensar, primero, que la memoria es un tipo de observación de segundo orden y, segundo, que el olvidar / recordar propio de ésta, implica la comparación. Si se acepta lo primero, sería erróneo llamar a la memoria social "función", mientras que lo segundo es difícil de sostener, ya que los conceptos de memoria y oscilación, tal como fueron descriptos más arriba, no implican la comparación y sería complicado encontrar un resquicio por donde incluir esta operación entre estos dos conceptos. Por último, y probablemente lo más importante, esta observación de segundo orden que incluye la comparación “copia en el plano de la observación de segundo orden” (2007a) estas descripciones. Este "copiar", hace volver, una vez más, hacia la metáfora del depósito, ya que esto supone la necesidad de almacenar lo copiado, para compararlo en un momento posterior. Por consiguiente, vuelve a aparecer la dependencia mnemónica respecto de otros sistemas.

\section{Conclusiones}

El trabajo ha tratado de ilustrar el concepto de memoria social de Luhmann, haciendo especial hincapié en la utilización de los conceptos tomados de las Laws of Form. Hennig (2000), por ejemplo, le ha criticado a Luhmann el uso de estos conceptos como algo meramente metafórico, juicio que se asemeja al de Maturana y Varela, respecto de la utilización que hace Luhmann de su categoría de autopoiesis (1994). Estos autores, abogando por un purismo conceptual, critican el "mal uso" de las nociones en cuestión. Pese a que puede llegar a sentirse la tentación de tratar a las formulaciones aquí reportadas como un simple uso metafórico de los conceptos de Spencer Brown, este camino sería demasiado perjudicial y cerraría la discusión en el punto en que debe comenzar. Por otro lado, tampoco se puede sostener alegremente que el sociólogo ha hecho un "uso" personal de los conceptos, sin importar demasiado de dónde extrajo tales instrumentos y cómo los ha utilizado. Esta vía también liquida la cuestión de manera prematura, sin llegar a mostrar la riqueza de la exégesis paralela de los dos textos.

No cabe duda de que Luhmann podría haberle ahorrado bastante trabajo al lector, de haber expresado de manera explícita la forma en que utilizaría los conceptos del matemático inglés (y de muchos otros autores). De todas maneras, el meollo de la cuestión que se juega en la relación Luhmann-Spencer Brown a propósito del tema de la memoria, no es tanto saber hasta qué punto el primero intenta "aplicar" o es fiel a las formulaciones del segundo, sino investigar qué consecuencias tiene este uso conceptual y qué resultados para la teoría de sistemas sociales esto conlleva. El punto de arranque es la re-entry en la conceptualización de ambos autores, ya que es ella la que desencadena la unresolvable indeterminacy, que debe ser solucionada por la postulación de imaginary states. El concepto de re-entry, en Luhmann, se encuentra explícitamente ligado al de autoobservación, y es esta última la que provoca la condición de irresoluble indeterminación, que desemboca en la creación de estados imaginarios, correspondientes a las autodescripciones. Sin lugar a duda, Luhmann, como se ve aquí, busca "traducir" al lenguaje de su teoría de sistemas, lo expuesto por Spencer Brown, e intentará hacer lo mismo con la dupla conceptual función memoria-oscilación. La decisión teórica de trazar la analogía entre autodescripciones y funciones de 
segundo o mayor grado (con una o más re-entries) y ubicar a la memoria sólo en este contexto, lleva a la irremediable conclusión de tratar a la memoria social como una mera memoria semántica, también denominada "cultura". La primera consecuencia de este gesto podría ser que las comunicaciones de primer orden no contarían con la posibilidad de recordar (a nivel de la memoria social), sino que todo se olvidaría. Sólo las autodescripciones contarían con el privilegio mnemónico. La segunda consecuencia, de mayor importancia aun, es que la memoria social, si se la entiende de esta manera, no se ocuparía en ningún momento de hacer posible, por ejemplo, que una conversación no se esté olvidando continuamente a sí misma. Consecuentemente, esta conceptualización falla u olvida dar cuenta del corazón mismo del problema de la memoria social, teniendo que confiar, irremediablemente, en los rendimientos mnemónicos de los sistemas psíquicos y/o neurofisiológicos. Por lo tanto, queda aún por resolver si hay o tiene sentido que haya una memoria operativa de las operaciones comunicativas. De aceptarse tal falta, cabría preguntarse por la clausura operativa de los sistemas sociales, puesto que esto podría implicar una dependencia informativa respecto del entorno.

Otro punto de importancia en relación con esta temática, es que Luhmann parece tomar el concepto specerbrowniano de memoria, porque este le aseguraría dejar atrás la metáfora del depósito, al permitirle tratar a la memoria como circuito. Sin embargo, el problema aquí es que se termina sustituyendo una metáfora por otra, ya que las enormes redes que teje la comunicación no pueden ser equiparables a un circuito circular y cerrado, tal como lo propone Spencer Brown. Para el matemático, la memoria es propia de ese circuito, de esa función que se despliega al empezar a oscilar. El problema, al querer transferir semánticamente estas conclusiones, es que la comunicación no parece referirse a una memoria central, donde converja todo aquello que debe ser recordado. La sociedad no es una gran y única conversación, ni tampoco una red comunicativa única, donde todos los actos comunicativos terminen entrelazándose necesariamente en una especie de círculo de círculos, según la figura hegeliana. Por otra parte, hay una diferencia cualitativa en los recuerdos, puesto que, para Spencer Brown, lo único que se recuerda es el estado anteriormente marcado, y estas opciones son siempre dos: marked y unmarked. En el ámbito social, al sostenerse que la memoria lo que recuerda son distinciones, la memoria debería guardar el recuerdo de cientos de miles de distinciones diferentes, por lo cual, la diferencia entre ambos ámbitos es insalvable. Esta situación no se soluciona al otorgarle una memoria a cada sistema funcional de la sociedad, ya que, quizás, ni aun concediéndole una a cada sistema de interacción, la analogía podría sostenerse.

Los más optimistas, en este punto, podrían decir que no hay metáfora, que hay una clara reelaboración, por parte de Luhmann, del concepto de memoria, manteniendo cierto lenguaje spencerbrowniano. No obstante, esto no aleja el problema central que subyace a lo dicho en el párrafo anterior, que es la imposibilidad de concebir a la memoria social como circuito, dejando atrás la metáfora del depósito, que parecía ser la intención primaria de Luhmann. El problema de tratar con una memoria que trabaja con distinciones, todas ellas diferentes es: ¿cómo es posible que emerja el recuerdo?, ¿cómo es posible recordar en la comunicación actual, distinciones anteriormente utilizadas? Entonces, siguiendo los pasos de Maxwell, a la hora de resolver el problema de la segunda ley de la termodinámica, no quedaría más que postular la existencia de un demonio, como indica irónicamente von Foerster. Este demonio de la memoria se encargaría de hacer recordar en una comunicación qué otras distinciones podrían utilizarse, o sea, que se encargaría de seleccionar. De no aceptarse tal solución, debería confiarse en la capacidad selectiva de los sistemas psíquicos, que recordarían aquellas distinciones almacenadas en su propia memoria, más allá de que estas sean un producto meramente social. Esto significa que, por más de que existan libros u otros medios de almacenamiento de distinciones, estas distinciones no pueden entrar a formar parte de la 
comunicación si no son evocadas por alguna memoria que las recuerde. Ya que la memoria social parece no tener ninguna operatividad propia que permita pensar que ella puede intervenir en la comunicación, haciendo que se seleccionen determinadas distinciones, ese rol no puede recaer más que en los sistemas psíquicos intervinientes, quienes pueden recordar lo que pueden recordar, y no lo que nunca leyeron o escucharon. De esta manera, quizás sea mejor seguir hablando de una memoria colectiva, más o menos homogénea, más o menos compartida por las conciencias.

\section{Notas}

(1) Este trabajo es fruto del trabajo desarrollado en el marco del proyecto UBACYT “Análisis sistémico de las transformaciones de la unidad selectiva de la comunicación a partir de la utilización de tecnologías de la información y de la comunicación" (UBACyT 20020090200022 - Res. (CS) № 1004/10). Una versión preliminar de este trabajo fue presentada en las VI Jornadas de Sociología de la UNLP, con el título de "La memoria social en la teoría de sistemas sociales", el 10/12/2010 (Departamento de Sociología - Facultad de Humanidades y Ciencias de la Educación - UNLP, ISBN 978-950-34-0693-9).

(2) Esto es graficado de la siguiente manera $f=\langle\ldots\{[(a) b] a\} b\rangle$, teniendo en cuenta que la expresión original era $[(a) b]$. Utilizamos los paréntesis para no tener que reproducir la complicada forma de graficar spencerbrowniana.

(3) 'Spencer Brown trata la 'oscilator function' únicamente en referencia con la diferenciación marked / unmarked en el contexto de ecuaciones de segundo orden (...) Para una teoría semántica de la memoria debemos extender el concepto del oscilar a toda distinción que se utilice para observar; también a aquellas situadas entre dos ítemes marcados como serían por ejemplo las de nobleza / pueblo, o castillo / iglesias, o catedrales / iglesias urbanas; en donde las distinciones doblemente marcadas presuponen a su vez un espacio-no-marcado, ya que observar sólo es posible dentro del mundo el cual siempre permanece sin marca" [La nota y los subrayados son de Luhmann] (2007b: 460).

\section{Bibliografía}

Dionisio Areopagita, Pseudo. 1981. Tutte le opere. Milán: Rusconi.

Esposito, E. 2002. Soziales Vergessen. Formen und Medien des Gedächtnisses der Gesellschaft. Frankfurt am Mein: Suhrkamp.

Foerster, H. von. 2003a. What is memory that it may have hindsight and foresight as well? En: H. von Foerster. Unterstanding understanding: essays on cybernetics and cognition. Nueva York: Springer, pp. 101131.

Foerster, H. von. 2003b. Objects: tokens for (eigen-) behaviors. En: H. von Foerster. Unterstanding understanding: essays on cybernetics and cognition. Nueva York: Springer, pp. 261-271.

Hennig, B. 2000. Luhmann und die Formale Mathematik. En: P.U. Merz-Benz y G. Wagner (Editores). Die Logik der Systeme. Zur Kritik der systemtheoretischen Soziologie Niklas Luhmanns. Konstanz: Universitätsverlag Konstanz, pp. 157-198.

Kandel, E. 2001. The molecular biology of memory storage: a dialog between genes and synapses. Bioscience 
Reports 21(5): 565-611.

Keys, J. 1974. Only two can play this game. Nueva York: Bantam Books.

Luhmann, N. 1984. Soziale Systeme. Grundriß einer allgemeinen Theorie. Frankfurt a.M.: Suhrkamp Verlag.

Luhmann, N. 1988. Frauen, Männer und George Spencer Brown. Zeitschrift für Soziologie 17: 47-71.

Luhmann, N. 1995. Why does society describe itself as postmodern? Cultural Critique 30: 171-186.

Luhmann, N. 1996. La ciencia de la sociedad. México: Anthropos.

Luhmann, N. 1997a. Globalization or world society: how to conceive of modern society? International Review of Sociology 7(1): 67-79.

Luhmann, N. 1997b. La cultura como concepto histórico. Historia y Grafía 8: 11-33.

Luhmann, N. 1998. Sistemas Sociales. Lineamientos para una teoría general. Barcelona: Anthropos.

Luhmann, N. 2000a. Die Politik der Gesellschaft. Frankfurt a.M.: Suhrkamp Verlag.

Luhmann, N. 2000b. Organisation und Entscheidung. Opladen / Wiesbaden: West deutscher Verlag GmbH.

Luhmann, N. 2002. Identity - what or how. En: N. Luhmann. Theories of distinction. Redescribing the description of modernity. Stanford: Stanford University Press, pp. 113-127.

Luhmann, N. 2005. El arte de la sociedad. México: Herder.

Luhmann, N. 2007a. La religión de la sociedad. Madrid: Trotta.

Luhmann, N. 2007b. La sociedad de la sociedad. México: Herder.

Luhmann, N. 2008. Eine Redeskription 'romantischer Kunst'. En: N. Luhmann (N. Werber editor). Schriften zu Kunst und Literatur. Frankfurt a.M.: Suhrkamp Verlag, pp. 353-371.

Maturana, H. y Varela, F. 1994. De máquinas y seres vivos. Santiago: Editorial Universitaria.

Spencer Brown, G. 1972. Laws of form. Nueva York: The Julian Press, Inc.

Recibido el 21 Jun 2011

Aceptado el 17 Sep 2011 